\title{
Clustering in complex directed networks
}

\author{
Giorgio Fagiolo* \\ Sant'Anna School of Advanced Studies, Laboratory of Economics and Management, Piazza Martiri della Libertà 33, I-56127 Pisa, Italy
}

(Received 6 February 2007; published 16 August 2007)

\begin{abstract}
Many empirical networks display an inherent tendency to cluster, i.e., to form circles of connected nodes. This feature is typically measured by the clustering coefficient (CC). The CC, originally introduced for binary, undirected graphs, has been recently generalized to weighted, undirected networks. Here we extend the $\mathrm{CC}$ to the case of (binary and weighted) directed networks and we compute its expected value for random graphs. We distinguish between CCs that count all directed triangles in the graph (independently of the direction of their edges) and CCs that only consider particular types of directed triangles (e.g., cycles). The main concepts are illustrated by employing empirical data on world-trade flows.
\end{abstract}

DOI: 10.1103/PhysRevE.76.026107

PACS number(s): 89.75.-k, 89.65.Gh, 87.23.Ge, 05.70.Ln

Networked structures emerge almost ubiquitously in complex systems. Examples include the Internet and the WWW, airline connections, scientific collaborations and citations, trade and labor-market contacts, friendship and other social relationships, business relations and research and development partnerships, cellular, ecological, and neural networks [1-3].

The majority of such "real-world" networks have been shown to display structural properties that are neither those of a random graph [4], nor those of regular lattices. For example, many empirically observed networks are small worlds $[5,6]$. These networks are simultaneously characterized by two features [7]. First, as happens for random graphs, their diameter [8] increases only logarithmically with the number of nodes. This means that, even if the network is very large, any two seemingly unrelated nodes can reach each other in a few steps. Second, as happens in lattices, small-world networks are highly clustered, i.e., any two neighbors of a given node have a probability of being themselves neighbors which is much larger than in random graphs.

Network clustering is a well-known concept in sociology, where notions such as "cliques" and "transitive triads" have been widely employed [9,10]. For example, friendship networks are typically highly clustered (i.e., they display high cliquishness) because any two friends of a person are very likely to be friends.

The tendency of a network to form tightly connected neighborhoods (more than in the random uncorrelated case) can be measured by the clustering coefficient (CC), see $[11,12]$. The idea is very simple. Consider a binary, undirected network (BUN) described by the graph $G=(N, A)$, where $N$ is the number of the nodes and $A=\left\{a_{i j}\right\}$ is the $N$ $\times N$ adjacency matrix, whose generic element $a_{i j}=1$ if and only if there is an edge connecting nodes $i$ and $j$ (i.e., if they are neighbors) and zero otherwise. Since the network is undirected, $A$ is symmetric [13]. For any given node $i$, let $d_{i}$ be its degree, i.e., the number of $i$ 's neighbors. The extent to which $i$ 's neighborhood is clustered can be measured by the percentage of pairs of $i$ 's neighbors that are themselves neighbors, i.e., by the ratio between the number of triangles in the graph $G$ with $i$ as one vertex (labeled as $t_{i}$ ) and the number of all possible triangles that $i$ could have formed [that is, $T_{i}=d_{i}\left(d_{i}-1\right) / 2$ ] [14]. It is easy to see that the CC for node $i$ in this case reads

$$
C_{i}(A)=\frac{\frac{1}{2} \sum_{j \neq i} \sum_{h \neq(i, j)} a_{i j} a_{i h} a_{j h}}{\frac{1}{2} d_{i}\left(d_{i}-1\right)}=\frac{\left(A^{3}\right)_{i i}}{d_{i}\left(d_{i}-1\right)},
$$

where $\left(A^{3}\right)_{i i}$ is the $i$ th element of the main diagonal of $A^{3}$ $=A A A$. Each product $a_{i j} a_{i h} a_{j h}$ is meant to count whether a triangle exists or not around $i$. Notice that the order of subscripts is irrelevant, as all entries in $A$ are symmetric. Of course, $C_{i} \in[0,1]$. The overall (network-wide) $\mathrm{CC}$ for the graph $G$ is then obtained by averaging $C_{i}$ over the $N$ nodes, i.e., $C=N^{-1} \sum_{i=1}^{N} C_{i}$. In the case of a random graph where each link is in place with probability $p \in(0,1)$, one has that $E[C]=p$ ( $E$ stands for the expectation operator).

Binary networks treat all edges present in $G$ as they were completely homogeneous. More recently, scholars have become increasingly aware of the fact that real networks exhibit a relevant heterogeneity in the capacity and intensity of their connections [15-20]. Allowing for this heterogeneity might be crucial to better understand the architecture of complex networks. In order to incorporate such a previously neglected feature, each edge $i j$ present in $G$ (i.e., such that $a_{i j}$ $=1$ ) is assigned a value $w_{i j}>0$ proportional to the weight of that link in the network. For example, weights can account for the amount of trade volumes exchanged between countries (as a fraction of their gross domestic product), the number of passengers traveling between airports, the traffic between two Internet nodes, the number of emails exchanged between pairs of individuals, etc. Without loss of generality, we can suppose that $w_{i j} \in[0,1][21]$. A weighted undirected network (WUN) is thus characterized by its $N \times N$ symmetric weight matrix $W=\left\{w_{i j}\right\}$, where $w_{i i}=0$, all $i$. Many network measures developed for BUNs have a direct counterpart in WUNs. For example, the concept of node degree can be replaced by that of node strength [15]:

$$
s_{i}=\sum_{j \neq i} w_{i j}
$$


For more complicated measures, however, extensions to WUNs are not straightforward. To generalize the CC of node $i$ to WUNs, one has indeed to take into account the weight associated to edges in the neighborhood of $i$. There are many ways to do that [22]. For example, suppose that a triangle $i h j$ is in place. One might then consider only weights of the edges $i h$ and $i j$ [15]. Alternatively, one might employ the weights of all the edges in the triangle. In turn, the total contribution of a triangle can be defined as the geometric mean of its weights [23] or simply as the product among them [24-27]. In what follows, we will focus on the extension of the CC to WUNs originally introduced in [23]:

$$
\tilde{C}_{i}(W)=\frac{(1 / 2) \sum_{j \neq i} \sum_{h \neq(i, j)} w_{i j}^{1 / 3} w_{i h}^{1 / 3} w_{j h}^{1 / 3}}{(1 / 2) d_{i}\left(d_{i}-1\right)}=\frac{\left(W^{[1 / 3]}\right)_{i i}^{3}}{d_{i}\left(d_{i}-1\right)},
$$

where we define $W^{[1 / k]}=\left\{w_{i j}^{1 / k}\right\}$, i.e., the matrix obtained from $W$ by taking the $k$ th root of each entry. As discussed in [22], the measure $\tilde{C}_{i}$ ranges in $[0,1]$ and reduces to $C_{i}$ when weights become binary. Furthermore, it takes into account weights of all edges in a triangle (but does not consider weights not participating in any triangle) and is invariant to weight permutation for one triangle. Notice that $\widetilde{C}_{i}=1$ only if the neighborhood of $i$ actually contains all possible triangles that can be formed and each edge participating in these triangles has unit (maximum) weight. Again, one can define the overall clustering coefficient for WUNs as $\widetilde{C}=N^{-1} \sum_{i=1}^{N} \widetilde{C}_{i}$.

In this paper we discuss extensions of the $\mathrm{CC}$ for BUNs and WUNs [Eqs. (1) and (3)] to the case of directed networks. It is well known that many real-world complex networks involve nonmutual relationships, which imply nonsymmetric adjacency or weight matrices. For instance, trade volumes between countries [28-30] are implicitly directional relations, as the export from country $i$ to country $j$ is typically different from the export from country $j$ to country $i$ (i.e., imports of $i$ from $j$ ). If such networks are symmetrized (e.g., by averaging imports and exports of country $i$ ), one could possibly underestimate important aspects of their network architecture.

Alternative extensions of the $\mathrm{CC}$ to weighted or directed networks have been recently introduced in the literature on "network motifs" [31]. As mentioned, [23] generalizes the $\mathrm{CC}$ to weighted-and possibly directed-networks. Similarly, [32] computes the recurrence of all types of three-node connected subgraphs in a variety of real-world binary directed networks from biochemistry, neurobiology, ecology, and engineering. However, the weighted CC in [23] does not explicitly discriminate between different directed triangles (cf. Fig. 1), while [32] does not allow for a weighted analysis. This work attempts to bridge the two latter approaches and presents a unifying framework where, in addition to the measures already discussed in [23,32], one is able to (i) explicitly account for directed and weighted links; and (ii) define a weighted, directed version of the $\mathrm{CC}$ for any type of triangle pattern (i.e., three-node connected subgraph). To compute such coefficients, we shall employ the actual and
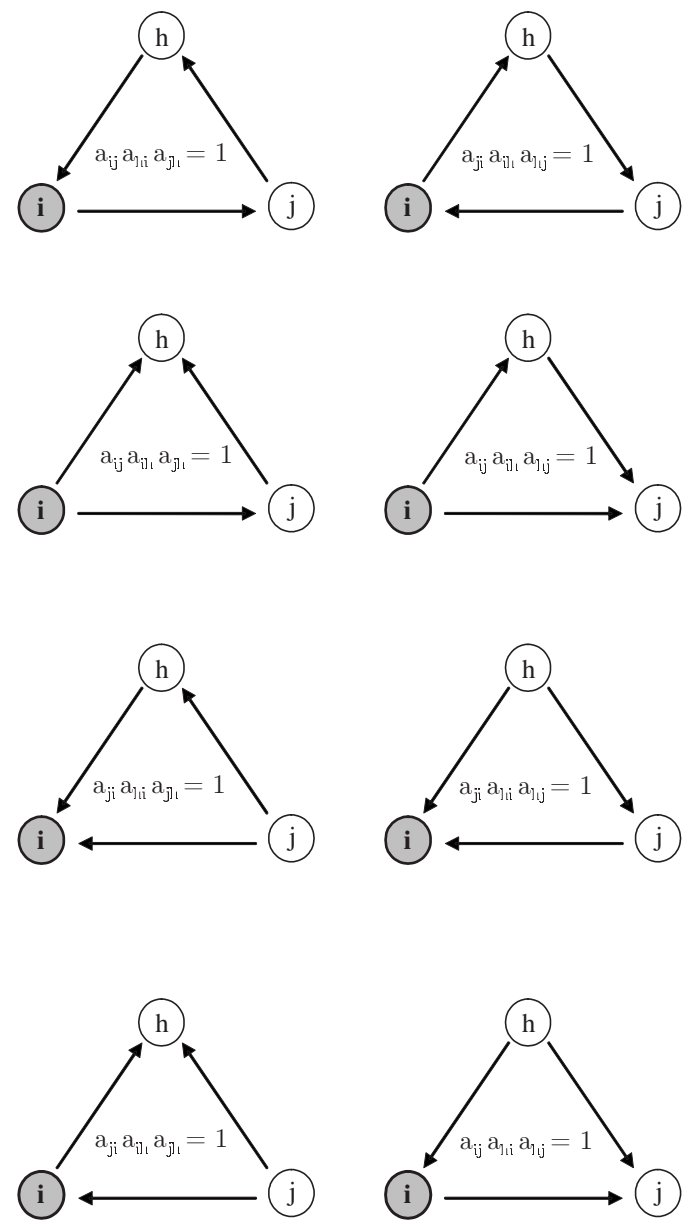

FIG. 1. Binary directed graphs. All eight different triangles with node $i$ as one vertex. Within each triangle is reported the product of the form $a_{* *} a_{* *} a_{* *}$ that works as indicator of that triangle in the network.

potential number of directed-triangle patterns of any given type.

Preliminaries. In directed networks, edges are oriented and neighboring relations are not necessarily symmetric. In the case of binary directed networks (BDNs), we define the in-degree of node $i$ as the number of edges pointing towards $i$ (i.e., inward edges). The out-degree of node $i$ is accordingly defined as the number of edges originating from $i$ (i.e., outward edges). Formally,

$$
\begin{gathered}
d_{i}^{\text {in }}=\sum_{j \neq i} a_{j i}=\left(A^{T}\right)_{i} \mathbf{1}, \\
d_{i}^{\text {out }}=\sum_{j \neq i} a_{i j}=(A)_{i} \mathbf{1},
\end{gathered}
$$

where $A^{T}$ is the transpose of $A,(A)_{i}$ stands for the $i$ th row of $A$, and $\mathbf{1}$ is the $N$-dimensional column vector $(1,1, \ldots, 1)^{T}$. The total degree of a node is simply the sum of its in- and out-degree: 


$$
d_{i}^{\text {tot }}=d_{i}^{\text {in }}+d_{i}^{\text {out }}=\left(A^{T}+A\right)_{i} \mathbf{1} .
$$

Finally, provided that no self-interactions are present, the number of bilateral edges between $i$ and its neighbors (i.e., the number of nodes $j$ for which both an edge $i \rightarrow j$ and an edge $j \rightarrow i$ exist) is computed as

$$
d_{i}^{\leftrightarrow}=\sum_{j \neq i} a_{i j} a_{j i}=A_{i i}^{2} .
$$

It is easy to see that in BUNs one has $d_{i}=d_{i}^{\text {tot }}-d_{i}^{\leftrightarrow}$.

The above measures can be easily extended to weighted directed networks (WDNs), by considering in-, out-, and total-strength [see Eq. (2)].

Binary directed networks. We begin by introducing the most general extension of the CC to BDNs, which considers all possible directed triangles formed by each node, no matter the directions of their edges. Consider node $i$. When edges are directed, $i$ can generate up to eight different triangles with any pair of neighbors [33]. Any product of the form $a_{i j} a_{i h} a_{j h}$ captures one particular triangle, see Fig. 1 for an illustration.

The CC for node $i\left(C_{i}^{D}\right)$ in BDNs can be thus defined (like in BUNs) as the ratio between all directed triangles actually formed by $i\left(t_{i}^{D}\right)$ and the number of all possible triangles that $i$ could form $\left(T_{i}^{D}\right)$. Therefore

$$
\begin{aligned}
C_{i}^{D}(A) & =\frac{t_{i}^{D}}{T_{i}^{D}}=\frac{(1 / 2) \sum_{j} \sum_{h}\left(a_{i j}+a_{j i}\right)\left(a_{i h}+a_{h i}\right)\left(a_{j h}+a_{h j}\right)}{\left[d_{i}^{\text {tot }}\left(d_{i}^{\text {tot }}-1\right)-2 d_{i}^{\leftrightarrow}\right]} \\
& =\frac{\left(A+A^{T}\right)_{i i}^{3}}{2\left[d_{i}^{\text {tot }}\left(d_{i}^{\text {tot }}-1\right)-2 d_{i}^{\hookleftarrow}\right]}
\end{aligned}
$$

where (also in what follows) sums span over $j \neq i$ and $h$ $\neq(i, j)$. In the first line of Eq. (8), the numerator of the fraction is equal to $t_{i}^{D}$, as it simply counts all possible products of the form $a_{i j} a_{i h} a_{j h}$ (cf. Fig. 1). To see that $T_{i}^{D}$ $=d_{i}^{\text {tot }}\left(d_{i}^{\text {tot }}-1\right)-2 d_{i}^{\leftrightarrow}$, notice that $i$ can be possibly linked to a maximum of $d_{i}^{\text {tot }}\left(d_{i}^{\text {tot }}-1\right) / 2$ pairs of neighbors and with each pair can form up to two triangles (as the edge between them can be oriented in two ways). This leaves us with $d_{i}^{\text {tot }}\left(d_{i}^{\text {tot }}\right.$ $-1)$ triangles. However, this number also counts "false" triangles formed by $i$ and by a pair of directed edges pointing to the same node, e.g., $i \rightarrow j$ and $j \rightarrow i$. There are $d_{i}^{\leftrightarrow}$ of such occurrences for node $i$, and for each of them we have wrongly counted two "false" triangles. Therefore by subtracting $2 d_{i}^{\leftrightarrow}$ from the above number we get $T_{i}^{D}$. This implies that $C_{i}^{D} \in[0,1]$. The overall CC for BDNs is defined as $C^{D}$ $=N^{-1} \sum_{i=1}^{N} C_{i}^{D}$.

The CC in Eq. (8) has two nice properties. First, if $A$ is symmetric, then $C_{i}^{D}(A)=C_{i}(A)$, i.e., it reduces to Eq. (1) when networks are undirected. To see this, note that if $A$ is symmetric then $d_{i}^{\text {tot }}=2 d_{i}$ and $d_{i}^{\leftrightarrow}=d_{i}$. Hence

$$
C_{i}^{D}(A)=\frac{(2 A)_{i i}^{3}}{2\left[2 d_{i}\left(2 d_{i}-1\right)-2 d_{i}\right]}=\frac{(A)_{i i}^{3}}{d_{i}\left(d_{i}-1\right)}=C_{i}(A) .
$$

Second, the expected value of $C_{i}^{D}$ in random graphs, where each edge is independently in place with probability $p \in(0,1)$ [i.e., $a_{i j}$ are i.i.d. Bernoulli $(p)$ random variables], is still $p$ (as happens for BUNs). Indeed, the expected value of $t_{i}^{D}$ is simply $4(N-1)(N-2) p^{3}$. Furthermore, note that $d_{i}^{i n}$ $\sim d_{i}^{\text {out }} \sim \operatorname{bin}(N-1, p) \quad$ and $\quad d_{i}^{\text {tot }} \sim \operatorname{bin}(2(N-1), p)$. Hence $E\left[d_{i}^{\text {tot }}\left(d_{i}^{\text {tot }}-1\right)\right]=E\left[d_{i}^{\text {tot }}\right]^{2}-E\left[d_{i}^{\text {tot }}\right]=2(N-1)(2 N-3) p^{2}$. Similarly, $E\left[d_{i}^{\leftrightarrow}\right]=(N-1) p^{2}$, which implies that $E\left[T_{i}^{D}\right]=4(N-1)$ $\times(N-2) p^{2}$ and finally that $E\left[C_{i}^{D}\right]=p$.

Weighted directed networks. The CC for BDNs defined above can be easily extended to weighted graphs by replacing the number of directed triangles actually formed by $i\left(t_{i}^{D}\right)$ with its weighted counterpart $\vec{t}_{i}^{D}$. Given Eq. (3), $\widetilde{t}_{i}^{D}$ can be thus computed by substituting $A$ with $W^{[1 / 3]}$. Hence

$$
\tilde{C}_{i}^{D}(W)=\frac{\tilde{t}_{i}^{D}}{T_{i}^{D}}=\frac{\left[W^{[1 / 3]}+\left(W^{T}\right)^{[1 / 3]}\right]_{i i}^{3}}{2\left[d_{i}^{t o t}\left(d_{i}^{t o t}-1\right)-2 d_{i}^{\hookrightarrow}\right]} .
$$

Note that when the graph is binary $(W=A)$, then $(W)^{[1 / 3]}$ $=W=A$. Hence $\widetilde{C}_{i}^{D}(A)=C_{i}^{D}(A)$. Moreover, if $W$ is a symmetric weight matrix, then the numerator of $\widetilde{C}_{i}^{D}(W)$ becomes $\left[2 W^{[1 / 3]}\right]^{3}$. By combining this result with the denominator in Eq. (9), one has that $\widetilde{C}_{i}^{D}(W)=\widetilde{C}_{i}(W)$ for any symmetric $W$ [34].

To compute expected values of $\widetilde{C}_{i}^{D}$ in random graphs, suppose that weights are drawn using the following two-step algorithm. First, assume that any directed edge $i \rightarrow j$ is in place with probability $p$ (independently across all possible directed edges). Second, let the weight $w_{i j}$ of any existing directed edge (i.e., in place after the first step) be drawn from an independent random variable uniformly distributed over $(0,1][35]$. In this case, one has that $E\left[w_{i j}\right]^{1 / 3}=\frac{3 p}{4}$. It easily follows that for this class of random weighted graphs:

$$
E\left[\widetilde{C}_{i}^{D}\right]=E\left[\widetilde{C}_{i}\right]=\left(\frac{3}{4}\right)^{3} p<p .
$$

The overall CC for WDN is again defined as $\tilde{C}^{D}$ $=N^{-1} \sum_{i=1}^{N} \widetilde{C}_{i}^{D}$.

Clustering and patterns of directed triangles. The CCs for BDNs and WDNs defined above treat all possible directed triangles as they were the same, i.e., if directions were irrelevant. In other words, both $C^{D}$ and $\widetilde{C}^{D}$ operate a symmetrization of the underlying directed graph in such a way that the original asymmetric adjacency (respectively, weight) matrix $A$ (respectively, $W$ ) is replaced by the symmetric matrix $A$ $+A^{T}$ [respectively, $\left.W^{[1 / 3]}+\left(W^{T}\right)^{[1 / 3]}\right]$. This means that in the transformed graph, all directed edges are now bilateral. Furthermore, in binary (respectively, weighted) graphs, edges that were already bilateral count as two (respectively, receive a weight equal to the sum of the weights of the two directed edges raised to $1 / 3$ ).

However, in directed graphs, triangles with edges pointing in different directions have a completely different interpretation in terms of the resulting flow pattern. To put it differently, they account for different network motifs. Looking again at Fig. 1, it is possible to single out four patterns of directed triangles from $i$ 's perspective. These are (i) cycle, when there exists a cyclical relation among $i$ and any two of its neighbors $(i \rightarrow j \rightarrow h \rightarrow i$, or vice versa); (ii) middleman, 
when one of $i$ 's neighbors (say $j$ ) both holds an outward edge to a third neighbor (say $h$ ) and uses $i$ as a medium to reach $h$ in two steps [36]; (iii) in, where $i$ holds two inward edges; and (iv) out, where $i$ holds two outward edges.

When one is interested in measuring clustering in directed networks, it is important to separately account for each of the above patterns. This can be done by building a $\mathrm{CC}$ for each pattern (in both BDNs and WDNs). As usual, each CC is defined as the ratio between the number of triangles of that pattern actually formed by $i$ and the total number of triangles of that pattern that $i$ can possibly form. Each CC will then convey information about clustering of each different pattern within tightly connected directed neighborhoods. In order to do that, we recall that the maximum number of all possible directed triangles that $i$ can form (irrespective of their pattern) can be decomposed as

$$
\begin{aligned}
T_{i}^{D}= & d_{i}^{\text {tot }}\left(d_{i}^{\text {tot }}-1\right)-2 d_{i}^{\leftrightarrow} \\
= & {\left[d_{i}^{\text {in }} d_{i}^{\text {out }}-d_{i}^{\leftrightarrow}\right]+\left[d_{i}^{\text {in }} d_{i}^{\text {out }}-d_{i}^{\leftrightarrow}\right]+\left[d_{i}^{\text {in }}\left(d_{i}^{\text {in }}-1\right)\right] } \\
& +\left[d_{i}^{\text {out }}\left(d_{i}^{\text {out }}-1\right)\right]=T_{1}^{D}+T_{2}^{D}+T_{3}^{D}+T_{4}^{D} .
\end{aligned}
$$

Let $\left\{T_{i}^{c y c}, T_{i}^{\text {mid }}, T_{i}^{\text {in }}, T_{i}^{\text {out }}\right\}$ be the maximum number of cycles, middlemen, ins, and outs that $i$ can form. Inspection suggests that $T_{i}^{\text {cyc }}=T_{1}^{D}, T_{i}^{\text {mid }}=T_{2}^{D}, T_{i}^{i n}=T_{3}^{D}$, and $T_{i}^{\text {out }}=T_{4}^{D}$. To see why, consider, for example, $T_{i}^{c y c}$. In that pattern type (see Fig. 1, top panels), node $i$ is characterized by one inward link and one outward link. The maximum number of such patterns is given by $d_{i}^{\text {in }} d_{i}^{\text {out }}$. Again, this also counts "false" triangles, formed by $i$ and by a pair of directed edges pointing to and from a same node $j$. Therefore one has to subtract $d_{i}^{\hookrightarrow}$ to get $T_{i}^{c y c}$. Incidentally, notice that $T_{i}^{c y c}=T_{i}^{\text {mid }}$. The reason why this is indeed the case becomes evident when one compares the top and the bottom pairs of triangle patterns in Fig. 1. Indeed, cycles and middlemen only differ from the orientation of the link connecting the partners of the reference node $(i)$, which does not affect the maximum number of triangles that $i$ can form.

In order to count all actual triangles formed by $i$, we notice that

$$
\begin{aligned}
t_{i}^{D}=\left(A+A^{T}\right)_{i i} & =\left(A^{3}\right)_{i i}+\left(A A^{T} A\right)_{i i}+\left(A^{T} A^{2}\right)_{i i}+\left(A^{2} A^{T}\right)_{i i} \\
& =t_{1}^{D}+t_{2}^{D}+t_{3}^{D}+t_{4}^{D} .
\end{aligned}
$$

By letting $\left\{t_{i}^{c y c}, t_{i}^{\text {mid }}, t_{i}^{\text {in }}, t_{i}^{\text {out }}\right\}$ be the actual number of cycles, middlemen, ins, and outs formed by $i$, simple algebra reveals that $t_{i}^{c y c}=t_{1}^{D}, t_{i}^{\text {mid }}=t_{2}^{D}, t_{i}^{\text {in }}=t_{3}^{D}$, and $t_{i}^{\text {out }}=t_{4}^{D}$. For example,

$$
\begin{aligned}
t_{i}^{c y c} & =\frac{1}{2} \sum_{j} \sum_{h}\left[a_{i j} a_{j h} a_{h i}+a_{i h} a_{h j} a_{j i}\right] \\
& =\frac{1}{2} A_{(i)} A^{T} A^{(i)}=A_{(i)} A A^{(i)}=A_{(i i)}^{3} .
\end{aligned}
$$

Similarly,

$$
\begin{aligned}
t_{i}^{\text {mid }} & =\frac{1}{2} \sum_{j} \sum_{h}\left[a_{i j} a_{h j} a_{h i}+a_{i h} a_{j h} a_{j i}\right] \\
& =\frac{1}{2}\left[A_{(i)}^{T} A\left(A^{T}\right)^{(i)}+A_{(i)} A^{T} A^{(i)}\right]=A_{(i)} A^{T} A^{(i)}=\left(A A^{T} A\right)_{(i i)} .
\end{aligned}
$$

Notice that although $T_{i}^{c y c}=T_{i}^{\text {mid }}$, now $t_{i}^{c y c} \neq t_{i}^{m i d}$ as long as $A$ is asymmetric.

Summing up, we can define a CC for each pattern as follows:

$$
C_{i}^{*}=\frac{t_{i}^{*}}{T_{i}^{*}},
$$

where $\{*\}=\{c y c$, mid, in out $\}$.

In the case of weighted networks, it is straightforward to replace $t_{i}^{*}$ with its weighted counterpart $\widetilde{t}_{i}^{*}$, where the adjacency matrix $A$ has been replaced by $W^{[1 / 3]}$. We then accordingly define

$$
\widetilde{C}_{i}^{*}=\frac{\widetilde{t}_{i}^{*}}{T_{i}^{*}},
$$

where $\{*\}=\{c y c$, mid, in out $\}$. To summarize the above discussion, we report in Table I a taxonomy of all possible triangles with related measures for BDNs and WDNs.

Two remarks on Eqs. (16) and (17) are in order. First, note that, for $\{*\}=\{c y c$, mid, in, out $\}$, (i) when $A$ is symmetric, $C_{i}^{*}=C_{i}$; (ii) when $W$ is binary, $\widetilde{C}_{i}^{*}=C_{i}^{*}$; (iii) when $W$ is symmetric, $\widetilde{C}_{i}^{*}=\widetilde{C}_{i}$. Second, in random graphs one still has that $E\left[C_{i}^{*}\right]=p$ and $E\left[\widetilde{C}_{i}^{*}\right]=\left(\frac{3}{4}\right)^{3} p$.

Finally, network-wide clustering coefficients $C^{*}$ and $\widetilde{C}^{*}$ can be built for any triangle pattern $\{c y c$, mid, in, out $\}$ by averaging individual coefficients over the $N$ nodes.

These aggregate coefficients can be employed to compare the relevance of, say, cyclelike clustering among different networks, but not to assess the relative importance of cyclelike and middlemenlike clustering within a single network. In order to perform within-network comparisons, one can instead compute the fraction of all triangles that belong to the pattern $\{*\} \in\{c y c$, mid, in, out $\}$ in $i$ 's neighborhood, that is,

$$
f_{i}^{*}=\frac{t_{i}^{*}}{t_{i}^{D}}, \quad \widetilde{f}_{i}^{*}=\frac{\widetilde{t}_{i}^{*}}{\widetilde{t}_{i}^{D}},
$$

and then averaging them out over all nodes. Since for $\{*\} \in\{c y c$, mid, in,$o u t\}$ we have that $\Sigma_{*} f_{i}^{*}=1$ and $\Sigma_{*} \tilde{f}_{i}^{*}=1$, the above coefficients can be used to measure the contribution of each single pattern to the overall clustering coefficient. Notice that, in the case of BDNs, $f_{i}^{*}$ coefficients simply recover the recurrence of each pattern in the network, as computed in [32].

Empirical application. The above concepts can be meaningfully illustrated in the case of the empirical network describing world trade among countries (i.e., the "world trade network," WTN in what follows). Source data are provided by [37] and records, for any given year, imports and exports 
TABLE I. A taxonomy of the patterns of directed triangles and their associated clustering coefficients. For each pattern, we show the graph associated to it, the expression that counts how many triangles of that pattern are actually present in the neighborhood of $i\left(t_{i}^{*}\right)$, the maximum number of such triangles that $i$ can form $\left(T_{i}^{*}\right)$, for $*=\{c y c$, mid, in ,out,$D\}$, and the associated clustering coefficients for BDNs and WDNs. Note that in the last column $\hat{W}=W^{[1 / 3]}=\left\{w_{i j}^{1 / 3}\right\}$.

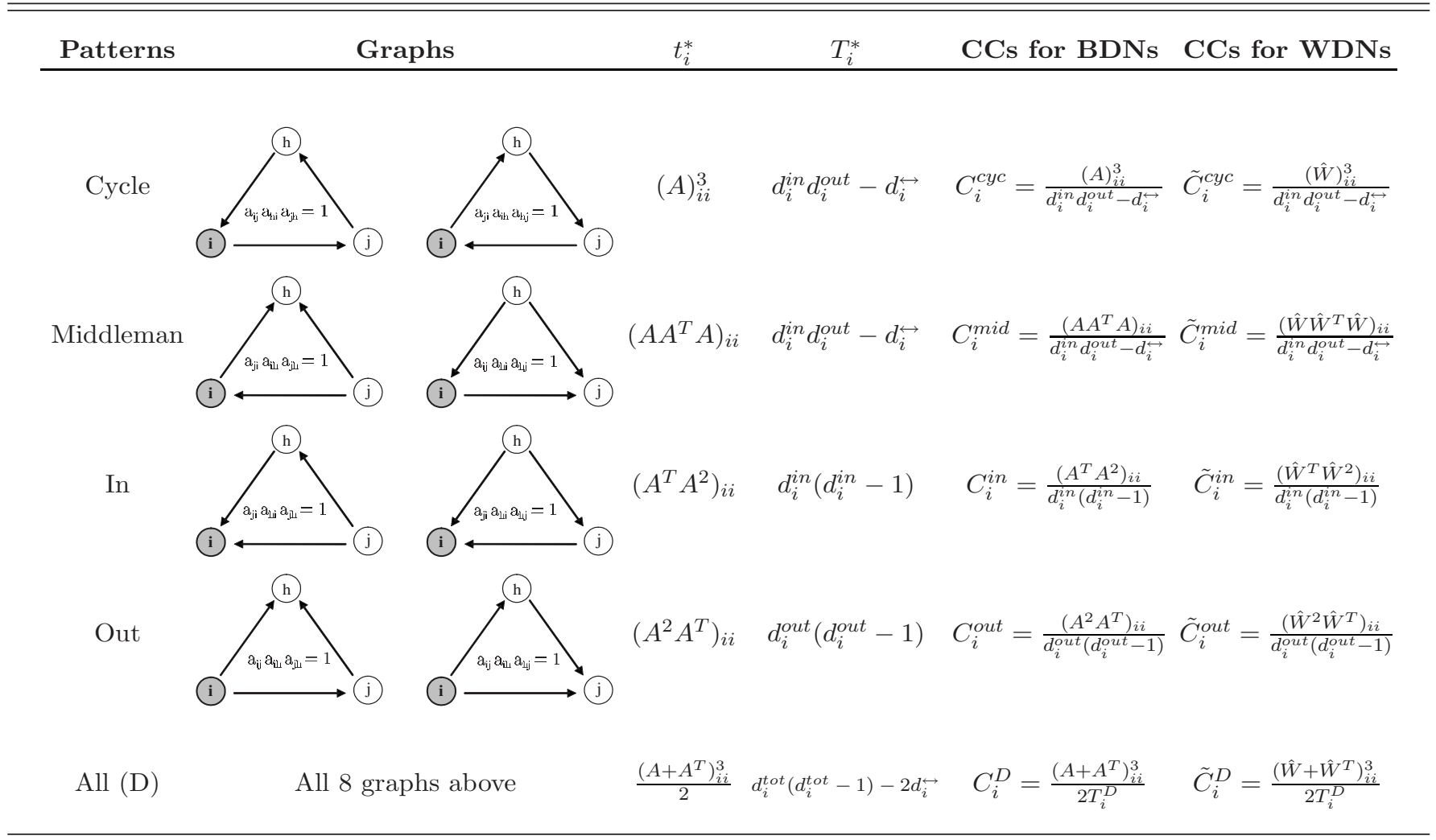

from and to a large sample of countries (all figures are expressed in current U.S. dollars). Here, for the sake of exposition, we focus on the year 2000 only [38]. We choose to build an edge between any two countries in the WTN if there is a nonzero trade between them and we assume that edge directions follow the flow of commodities. Let $x_{i j}$ be $i$ 's exports to country $j$ and $m_{j i}$ be imports of $j$ from $i$. In principle, $x_{i j}=m_{j i}$. Unfortunately, due to measurement problems, this is not the case in the database. In order to minimize this prob-

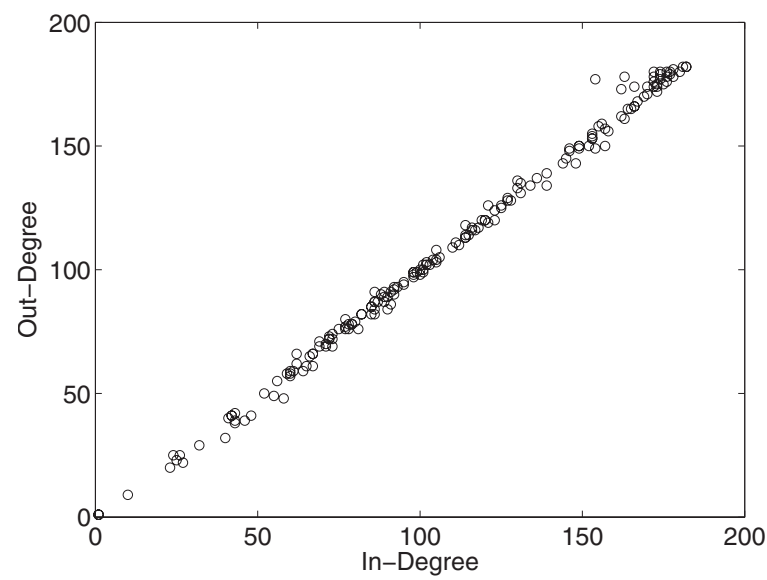

FIG. 2. WTN: In- vs out-degree in the binary case. Axes are in $\log _{10}$ scale. lem, we will focus here on "adjusted exports" defined as $e_{i j}=\left(x_{i j}+m_{j i}\right) / 2$ and we build a directed edge from country $i$ to country $j$ if and only if country $i$ 's adjusted exports to country $j$ are positive. Thus the generic entry of the adjacency matrix $a_{i j}$ is equal to 1 if and only if $e_{i j}>0$ (and 0 otherwise). Notice that, in general, $e_{i j} \neq e_{j i}$. In order to weight edges, adjusted exports can be tentatively employed. However, exporting levels are trivially correlated with the "size" of exporting and importing countries, as measured,

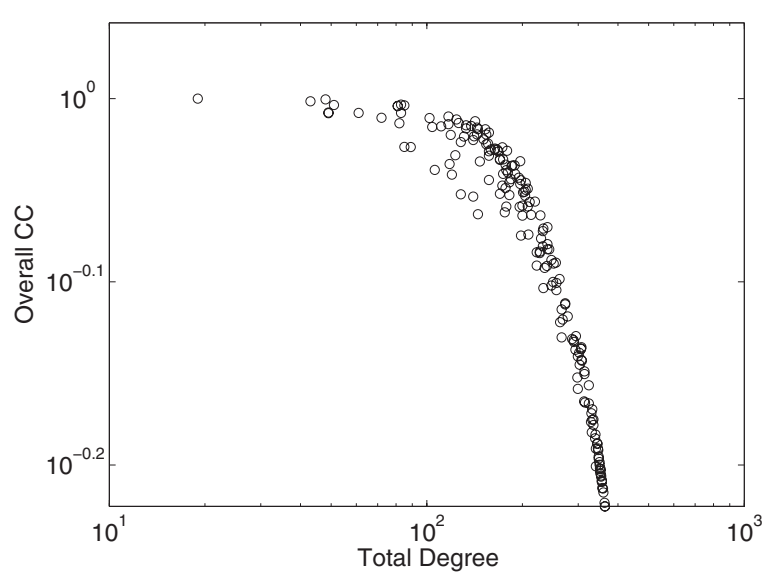

FIG. 3. WTN: Overall directed clustering coefficient vs totaldegree in the binary case. Axes are in $\log _{10}$ scale. 


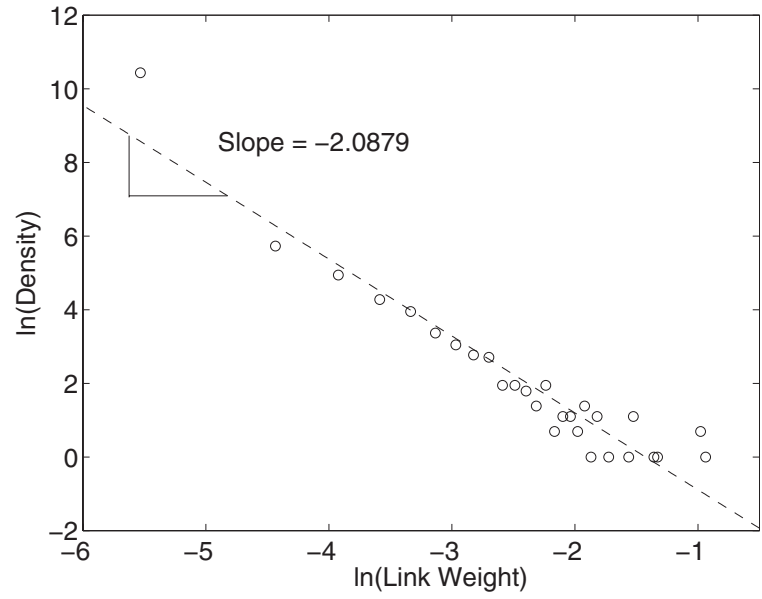

FIG. 4. WTN: Log-log plot of the weight distribution.

e.g., by their gross domestic products (GDPs). To avoid such a problem, we first assign each existing edge a weight equal to $\widetilde{w}_{i j}=e_{i j} / G D P_{i}$, where $G D P_{i}$ is country $i$ 's GDP expressed in 2000 U.S. dollars. Second, we define the actual weight matrix as

$$
W=\left\{w_{i j}\right\}=\frac{\widetilde{w}_{i j}}{\max _{h, k=1}^{N}\left\{\widetilde{w}_{h k}\right\}},
$$

to have weights in the unit interval. Each entry $w_{i j}$ tells us the extent to which country $i$ (as a seller) depends on $j$ (as a buyer). The out-strength of country $i$ (i.e., $i$ 's exports-to-GDP ratio) will then measure how $i$ (as a seller) depends on the rest of the world (as a buyer). Similarly, in-strengths denote how dependent is the rest of the world on $i$ (as a buyer) [39].

The resulting WTN comprises $N=187$ nodes/countries and 20105 directed edges. The density is therefore very high $(\delta=0.5780)$. As expected, the binary WTN is substantially symmetric: there is a 0.9978 correlation [40] between in- and out-degree (see Fig. 2) and the (nonscaled) $S$ measure introduced in [41] is close to zero (0.003 97), indicating that the underlying binary graph is almost undirected.

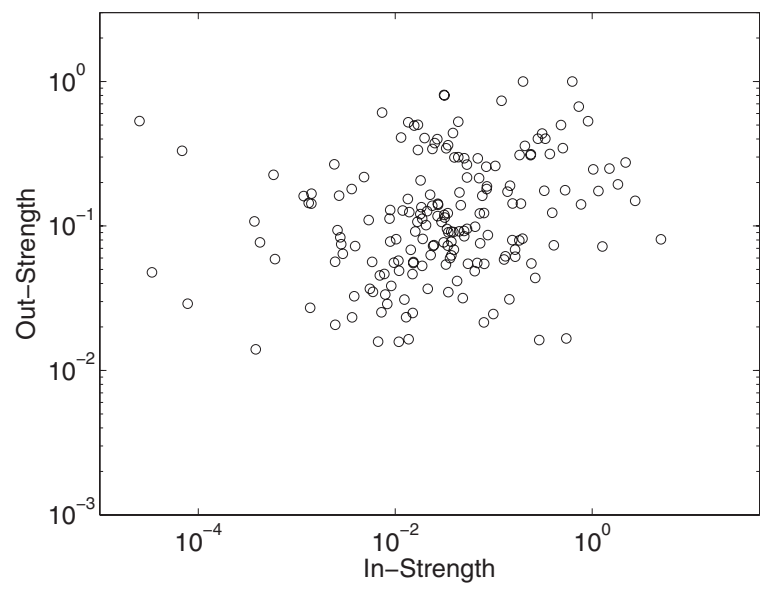

FIG. 5. WTN: In-strength vs out-strength. Axes are in $\log _{10}$ scale.

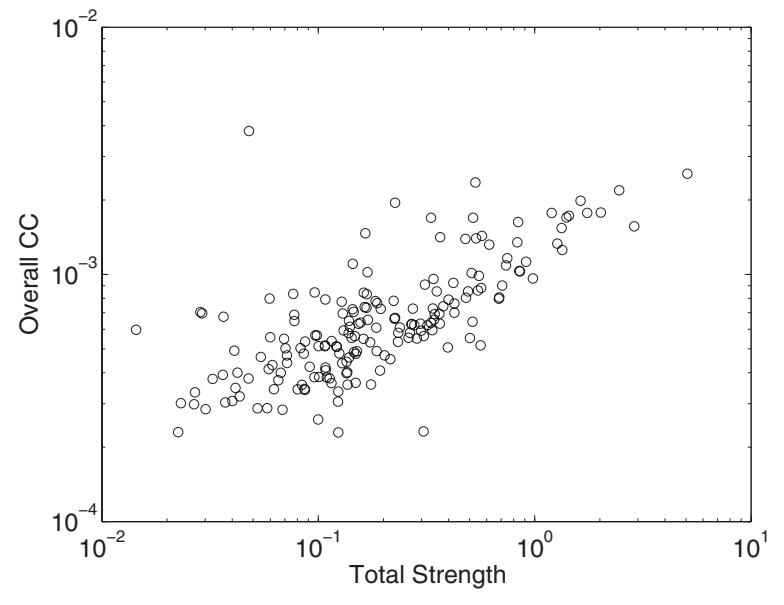

FIG. 6. WTN: Overall $\mathrm{CC}$ vs total strength in the WDN case. Axes are in $\log _{10}$ scale.

Thus, in the binary case, there seems to be no value added in performing a directed analysis: since $A$ is almost symmetric, we should not detect any significant differences among clustering measures for our four directed triangle patterns. Indeed, we find that $C^{D}=0.8125$, while $C^{c y c}=0.8123, C^{\text {mid }}$ $=0.8127, C^{\text {in }}=0.8142, C^{\text {out }}=0.8108$ [42]. The fact that $C^{D}$ $>\delta$ also indicates that the binary (directed) WTN is more clustered than it would be if it were random (with density $\delta=0.5780)$. Finally, Fig 3 shows that individual CCs $\left(C_{i}^{D}\right)$ are negatively correlated with total degree $\left(d_{i}^{\text {tot }}\right)$, the correlation coefficient being -0.4102 . This implies that countries with few (respectively, many) partners tend to form very (respectively, poorly) connected clusters of trade relationships.

The binary network does not take into account the heterogeneity of export flows carried by edges in the WTN. Indeed, when one performs a WDN analysis on the WTN, the picture changes completely. To begin with, note that weights $w_{i j}$ are on average very weak (0.0009) but quite heterogeneous (weight standard deviation is 0.0073 ). In fact, weight distribution is very skewed and displays a characteristic powerlaw shape (see Fig. 4) with a slope around -2 .

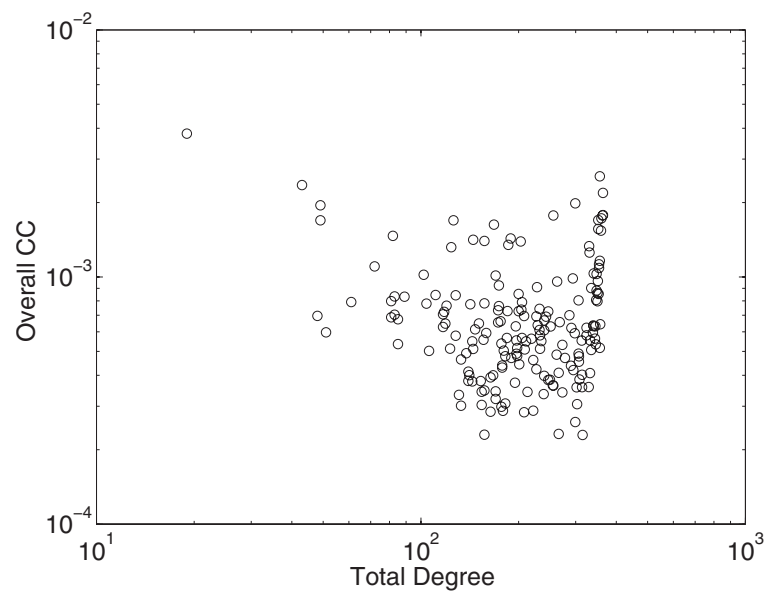

FIG. 7. WTN: Overall CC vs total degree in the WDN case. Axes are in $\log _{10}$ scale. 


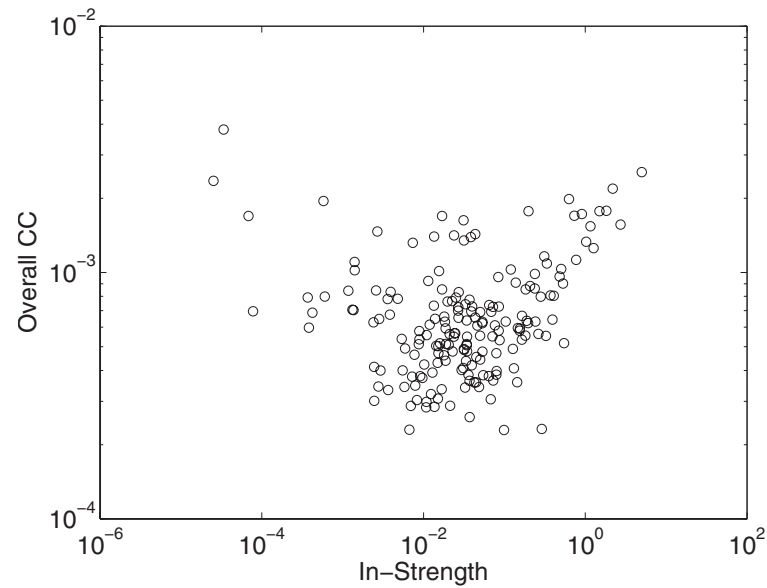

FIG. 8. WTN: Overall CC vs in-strength in the WDN case. Axes are in $\log _{10}$ scale.

The matrix $W$ is now weakly asymmetric. As Fig. 5 shows, in- and out-strengths are almost not correlated: the correlation coefficient is 0.09 (not significantly different from zero). Nevertheless, the (not-scaled) $S$ measure is still very low (0.1118), suggesting that an undirected analysis would still be appropriate. We will see now that, even in this borderline case, a weighted directed analysis of CCs provides a picture which is much more precise than (and sometimes at odds with) that emerging in the binary case.

First, unlike in the binary case, the overall average $\mathrm{CC}$ $\left(\widetilde{C}^{D}\right)$ is now very low $(0.0007)$ and significantly smaller than its expected value $(0.2438)$ in random graphs (with the same density $\delta=0.5780$, but independently, uniformly distributed weights). Notice, however, that $\widetilde{C}^{D}$ is almost equal to its expected value in directed graphs characterized by the same topology (as defined by the adjacency matrix A) but the same weight distribution (as defined by the nonzero elements in $W$ ), which turns out to be equal to 0.0005 (with a standard deviation of 0.0001) [43].

Second, $\tilde{C}_{i}^{D}$ is now positively correlated with total strength (the correlation coefficient is 0.6421), cf. Fig. 6. This means that, when weight heterogeneity is taken into account, the implication we have drawn in the binary case is reversed: countries that are more strongly connected tend to form more strongly connected trade circles. Indeed, $\widetilde{C}_{i}^{D}$ exhibits an almost null correlation with total degree, see Fig. 7.

Third, despite that the weighted network is only weakly asymmetric, there is a substantial difference in the way clustering is coupled with exports and imports. $\widetilde{C}_{i}^{D}$ is almost uncorrelated with in-strength (Fig. 8), while a positive slope is still in place when $\widetilde{C}_{i}^{D}$ is plotted against out-strength. Hence the low clustering level of weakly connected countries seems to depend mainly on their weakly exporting relationships.

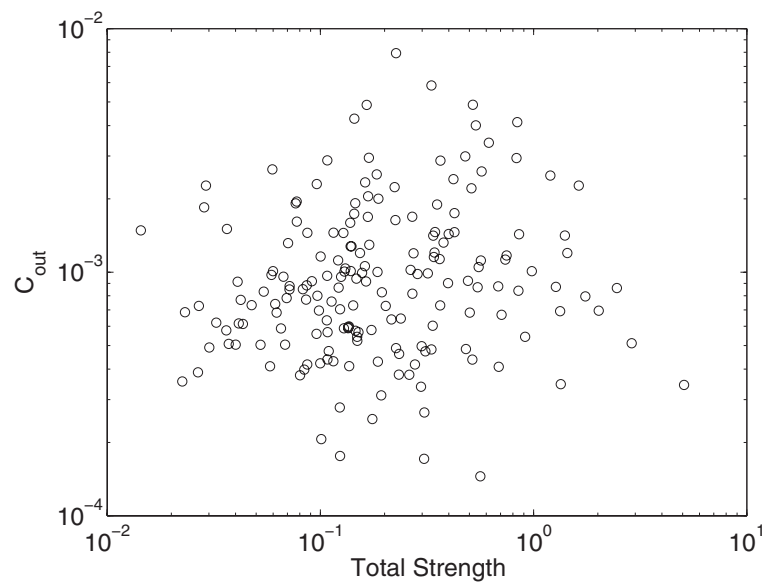

FIG. 9. WTN: $\tilde{C}_{i}^{\text {out }}$ vs total strength in the WDN case. Axes are in $\log _{10}$ scale.

Fourth, weighted $\mathrm{CC}$ coefficients associated to different triangle patterns now show a relevant heterogeneity: $\widetilde{C}^{*}$ range from 0.0004 (cycles) to 0.0013 (out). In addition, cycles only account for $18 \%$ of all triangles, while the other three patterns account for about $27 \%$ each. Therefore countries tend to form less frequently trade cycles, possibly because they involve economic redundancies.

Finally, CCs for different triangle patterns correlate with strength measures in different ways. While $\widetilde{C}_{i}^{c y c}, \widetilde{C}_{i}^{\text {mid }}$, and $\widetilde{C}_{i}^{i n}$ are positive and strongly correlated with total strength, $\widetilde{C}_{i}^{\text {out }}$ is not; see Fig. 9: countries tend to maintain exporting relationships with connected pairs of partners independently of the total strength of their trade circles.

Concluding remarks. In this paper, we have extended the clustering coefficient (CC), originally proposed for binary and weighted undirected graphs, to directed networks. We have introduced different versions of the $\mathrm{CC}$ for both binary and weighted networks. These coefficients count the number of triangles in the neighborhood of any node independently of their actual pattern of directed edges. In order to take edge directionality fully into account, we have defined specific $\mathrm{CCs}$ for each particular directed triangle pattern (cycles, middlemen, ins, and outs). For any $\mathrm{CC}$, we have also provided its expected value in random graphs. Finally, we have illustrated the use of directed CCs by employing world trade network (WTN) data. Our exercises show that directed CCs can describe the main clustering features of the underlying WTN's architecture much better than their undirected counterparts.

Thanks to Javier Reyes, Stefano Schiavo, and an anonymous referee, for their helpful comments. 
[1] R. Albert and A. Barabási, Rev. Mod. Phys. 74, 47 (2002).

[2] M. Newman, SIAM Rev. 45, 167 (2003).

[3] S. Dorogovtsev and J. Mendes, Evolution of Networks: From Biological Nets to the Internet and WWW (Oxford University Press, Oxford, 2003).

[4] B. Bollobás, Random Graphs (Academic Press, New York, 1985).

[5] The Small World, edited by M. Kochen (Ablex, Norwood, 1989).

[6] D. Watts, Small Worlds (Princeton University Press, Princeton, 1999).

[7] L. Amaral, A. Scala, M. Barthélemy, and H. Stanley, Proc. Natl. Acad. Sci. U.S.A. 97, 11149 (2000).

[8] As computed by the average shortest distance between any two nodes [44].

[9] S. Wasserman and K. Faust, Social Network Analysis. Methods and Applications (Cambridge University Press, Cambridge, England, 1994).

[10] J. Scott, Social Network Analysis: A Handbook (Sage, London, 2000).

[11] D. Watts and S. Strogatz, Nature (London) 393, 440 (1998).

[12] G. Szabó, M. Alava, and J. Kertész, in Complex Networks, Vol. 650 of Lecture Notes in Physics, edited by E. Ben-Naim, P. Krapivsky, and S. Redner (Springer, Berlin, 2004), pp. 139162.

[13] We also suppose that self-interactions are not allowed, i.e., $a_{i i}=0$, all $i$.

[14] From now on we will assume that the denominators of CCs are well defined. If not, we will simply set the CC to 0 .

[15] A. Barrat, M. Barthélemy, R. Pastor-Satorras, and A. Vespignani, Proc. Natl. Acad. Sci. U.S.A. 101, 3747 (2004).

[16] A. Barrat, M. Barthélemy, and A. Vespignani, Phys. Rev. Lett. 92, 228701 (2004).

[17] M. Barthélemy, A. Barrat, R. Pastor-Satorras, and A. Vespignani, Physica A 346, 34 (2005).

[18] A. DeMontis, M. Barthélemy, A. Chessa, and A. Vespignani, Environ. Plan. B: Plan. Des. (to be published).

[19] G. Kossinets and D. Watts, Science 311, 88 (2006).

[20] J. Onnela, J. Saramäki, J. Hyvönen, G. Szabó, M. Argollo de Menezes, K. Kaski, A. Barabási, and S. Kertész, New. J. Phys. 9, 179 (2007).

[21] If some $w_{i j}>1$, one can divide all weights by $\max _{i, j}\left\{w_{i j}\right\}$.

[22] J. Saramaki, M. Kivelä, J. Onnela, K. Kaski, and J. Kertész, Phys. Rev. E 75, 027105 (2007).

[23] J. P. Onnela, J. Saramaki, J. Kertész, and K. Kaski, Phys. Rev. E 71, 065103(R) (2005).

[24] P. Holme, S. Park, B. Kim, and C. Edling, Physica A 373, 821 (2007).

[25] B. Zhang and S. Hovarth, Stat. Appl. Genet. Mol. Biol. 4, (1), article 17 (2005), available online at http://www.bepress.com/ sagmb/vol4/iss1/art17.

[26] P. Grindrod, Phys. Rev. E 66, 066702 (2002).

[27] S. Ahnert, D. Garlaschelli, T. Fink, and G. Caldarelli, Phys. Rev. E 76, 016101 (2007).

[28] D. Garlaschelli and M. I. Loffredo, Phys. Rev. Lett. 93,
188701 (2004).

[29] M. A. Serrano and M. Boguñá, Phys. Rev. E 68, 015101(R) (2003).

[30] D. Garlaschelli and M. Loffredo, Physica A 355, 138 (2005).

[31] That is, sets of topologically equivalent subgraphs of a network.

[32] R. Milo, S. Shen-Orr, S. Itzkovitz, N. Kashtan, D. Chklovskii, and U. Alon, Science 298, 824 (2002).

[33] Of course, by a symmetry argument, they actually reduce to four different distinct patterns (e.g., those in the first column). We will keep the classification in eight types for the sake of exposition.

[34] The CC in Eq. (10) is similar to that presented by [22,23] but takes explicitly into account edge directionality in computing the maximum number of directed triangles $\left(T_{i}^{D}\right)$. Conversely, $[22,23]$ set $T_{i}^{D}=d_{i}\left(d_{i}-1\right)$.

[35] That is, $w_{i j}$ is a random variable equal to zero with probability $1-p$ and equal to a $U(0,1]$ with probability $p$. Of course this admittedly naïve assumption is made for mathematical convenience to benchmark our results in a setup where one is completely ignorant about the true (observed) weight distribution. In empirical applications, one would hardly expect observed weights to follow such a trivial distribution and more realistic assumptions should be made. For example, the expected value of CCs might be computed by bootstrapping (i.e., reshuffling) the observed empirical distribution of weights in $W$ across the same topological graph structure, as defined by the observed adjacency matrix $A$. See also below.

[36] These patterns can be also labeled as "broken" cycles, where the two neighbors whom $i$ attempts to build a cycle with actually invert the direction of the flow.

[37] K. Gleditsch, J. Conflict Resolut. 46, 712 (2002), available online at http://ibs.colorado.edu/ ksg/trade/

[38] That is, the most recent year available in the database. This also allows us to keep our discussion similar to that in [22].

[39] Dividing by $G D P_{j}$ would of course require a complementary analysis. Notice also that [22] defines adjusted exports as $e(i, j)=e(j, i)=[x(i, j)+m(j, i)+x(j, i)+m(i, j)] / 2$ thus obtaining an undirected binary or weighted network by construction.

[40] Here and in what follows, by correlation (or correlation coefficient) between two variables $X$ and $Y$, we mean the Spearman product-moment sample correlation, defined as $\sum_{i}\left(x_{i}-\bar{x}\right)\left(y_{i}\right.$ $-\bar{y}) /\left[(N-1) s_{X} s_{Y}\right]$, where $s_{X}$ and $s_{Y}$ are sample standard deviations. All correlation coefficients have been computed on original (linear) data, albeit log-log plots (in base 10) are sometime displayed.

[41] G. Fagiolo, Econ. Bull. 3, 1 (2007).

[42] Accordingly, one has that $f_{i}^{c y c}=0.2499, f_{i}^{\text {mid }}=0.2501, f_{i}^{\text {in }}$ $=0.2531$, and $f_{i}^{\text {out }}=0.2469$.

[43] To compute such expected values, we randomly reshuffled WTN weights in $W$ (by keeping $A$ fixed) and computed averages/standard deviations of CCs over $M=10000$ independent replications.

[44] Algorithms and Complexity, edited by J. van Leeuwen, Handbook of Theoretical Computer Science Vol. A (Elsevier, Amsterdam, 1999). 